\title{
A case report of primary signet ring cell carcinoma of the lung: imaging study and literature review
}

\author{
Mingguang Yang ${ }^{1, \#}$, Yanli Yang ${ }^{1 \#}$, Jing Chen ${ }^{3}$, Giulia M. Stella ${ }^{4}$, Sang-Won Um ${ }^{5}$, Yasmeen K. Tandon ${ }^{6}$, \\ Heng Liu ${ }^{1}$
}

${ }^{1}$ Department of Radiology, The Affiliated Hospital of Zunyi Medical University, Zunyi, China; ${ }^{2}$ Medical Imaging Department, Chongqing University Central Hospital, Chongqing, China; ${ }^{3}$ Department of Pathology, Chongqing University Central Hospital, Chongqing, China; ${ }^{4}$ Department of Medical Sciences and Infective Diseases, Unit of Respiratory Diseases, IRCCS Policlinico San Matteo Foundation and University of Pavia Medical School, Pavia, Italy; ${ }^{5}$ Division of Pulmonary and Critical Care Medicine, Department of Medicine, Samsung Medical Center, Sungkyunkwan University School of Medicine, Seoul, Korea; ${ }^{6}$ Department of Radiology, Mayo Clinic, Rochester, MN, USA

\#These authors contributed equally to this work.

Correspondence to: Dr. Heng Liu. Department of Radiology, The Affiliated Hospital of Zunyi Medical University, Medical Imaging Center of Guizhou Province, 149 Dalian Road, Huichuan District, Zunyi 563000, China. Email: zmcliuh@163.com.

\begin{abstract}
Signet ring cell carcinoma (SRCC) is a subtype of adenocarcinoma with characteristics of strong invasion and a poor prognosis. While it can occur in various organs, including the stomach, colon, esophagus, bladder, prostate, pancreas, and breast, primary lung SRCC is rare, and most SRCC found there are from gastrointestinal metastasis. Reports on primary lung SRCC are few and the aim of this study is to describe the imaging, histopathological, and immunohistochemical characteristics of a case of primary lung SRCC in our hospital. A 68-year-old female with no smoking history was admitted with recurrent cough, chest pain, and dyspnea of 2 months duration. Computed tomographic (CT) chest showed multiple solids nodules of different sizes and mass in the left upper lobe, lower lobe, and subpleural region. Multiple enlarged lymph nodes were seen in the mediastinum and left hilum. The aim of this paper is to improve the understanding of this tumor. A literature review identified 15 cases of primary lung SRCC with available CT imaging. Except for two patients with multiple ground glass nodules and multiple small nodules, the rest were solid, and ranged in size from 1.0 to $8 \mathrm{~cm}$. Only one patient had a cavity in the solid lesion. Immunohistochemical stains for thyroid transcription factor-1 (TTF-1) (13/13) and CK7 (12/12) showed positive reaction in all cases evaluated, and napsin A (3/4) were also positive, while all cases including CK20 (12/12) and CDX2 (6/6) were negative.
\end{abstract}

Keywords: Case report; lung; signet ring cell carcinoma (SRCC)

Submitted Jun 21, 2021. Accepted for publication Sep 15, 2021.

doi: $10.21037 /$ tlcr-21-654

View this article at: https://dx.doi.org/10.21037/tlcr-21-654

\section{Introduction}

Signet ring cell carcinoma (SRCC) is a special type of mucinous secretory adenocarcinoma, which is rich in intracellular mucins and pushes the nucleus to one side of the cell (1). Cytology smear (bronchial brushings) of the lung SRCC showed the cytomorphological characteristics of tumor Cells with delicate, vacuolated cytoplasm and eccentric nuclei, resembling alveolar macrophages (2). SRCC is derived from undifferentiated stem cells in the lamina propria of mucosa and has the characteristics of poor differentiation, diffuse infiltration, rapid growth, and difficult early diagnosis. Once SRCC infiltrates into the submucosa, signet ring cells (SRCs) will spread widely and form distant metastasis, making the tumor is highly invasive and carrying a poor prognosis. Primary SRCC usually occurs in the gastrointestinal tract but may also manifest in the lung, pancreas, breast, and bladder (3). Primary lung SRCC is an extremely rare subtype of lung adenocarcinoma, 
While Kish et al. (1) first reported 5 SRCC of lung tissue $(0.14 \%)$ out of 3,500 lung adenocarcinoma patients in 1989 , present literature on the tumor is mostly in the form of case reports (4) or small-scale retrospective clinical analysis (5), and information concerning the standardized clinical diagnosis, treatment, and prognosis of primary lung SRCC is scarce. In 2021, Cai et al. (6) performed a large population-based cohort analysis of database included in the Surveillance, Epidemiology, and End Results (SEER) registry from 2001 to 2015, compared with patients with lung mucinous adenocarcinoma, the clinical features of primary lung SRCC tended to had a lower age of onset, the average age at the time of diagnosis was 63.8 years. SRCC patients were significantly more likely to had a higher distant-stage disease (72.1\%), a higher pathological grade, a higher proportion of undergone chemotherapy (62.1\%). Prognostic factors included patient age, tumor grade, primary tumor site, summary stage, $\mathrm{T}$ stage, surgery, and chemotherapy, independent factors associated with a favorable patient prognosis included a primary site in the middle or lower lung lobe, underwent surgery, and underwent chemotherapy. However, age $\geq 80$ years, higher grade, distant summary stage disease, and $\mathrm{T} 4$ stage disease were linked to poor prognosis. In addition, the results of Iwasaki et al. (7) suggest that the proportion of SRCC components is a potential prognostic factor of primary lung adenocarcinoma.

We present the following article in accordance with the CARE reporting checklist (available at https://dx.doi. org/10.21037/tlcr-21-654).

\section{Case presentation}

A 68-year-old female with no smoking history was admitted with recurrent cough, chest pain, and dyspnea of 2 months duration. Computed tomographic (CT) chest image on the axial plane of the mediastinal window showed multiple nodules of different sizes and mass like soft tissue density in the left upper lobe, lower lobe, and subpleural region. Multiple enlarged lymph nodes were seen in the mediastinum and left hilum (Figure 1), while contrast-enhanced CT scan of the abdomen showed no obvious abnormality. Biopsy was compatible malignancy with SRC characteristics. Esophagogastroenteroscopy was performed to rule out primary intestinal origin tumor, and combined with $\mathrm{HE}$ and immunohistochemical results, the diagnosis of primary lung SRCC was confirmed. Immunohistochemical results were as follows: $\mathrm{CK}(+)$,
CK7(+), CK20(-), TTF1(+), P40(-), CDX2(-), Villin(-), S100(-), HMB45(-) and CEA(+), and Ki67 (labeling $=60 \%$ ) (Figure 2). Next-generation sequencing (NGS) studies were used to further identify adenocarcinoma, and seven diseaserelated genes including ALK, BRAF, EGFR, ERBB2, MET, $R E T$, and ROS1 were detected, while the $A L K$ fusion gene was negative. The patient received palliative treatment including traditional Chinese medicine, however, a repeat chest CT 3 months later showed that the pulmonary lesions were significantly enlarged and increased in number. The failure of treatment to reduce the burden of disease resulted in the patient opting for hospice care and later dying of her illness.

All procedures performed in studies involving human participants were in accordance with the ethical standards of the institutional and/or national research committee(s) and with the Helsinki Declaration (as revised in 2013). Written informed consent was obtained from the patient for publication of this case report and accompanying images. A copy of the written consent is available for review by the editorial office of this journal.

\section{Literature review}

The PubMed, Web of Science and Ovid databases were searched for English-language case reports and case series of primary lung SRCC published from 1989 to 2021. The keywords signet ring cell; lung or pulmonary; and carcinoma or adenocarcinoma were used. The flow chart of the literature selection process shows that 15 cases that had associated CT imaging (Figure 3). For each case, the first author, publication year and country, as well as the patient's age, gender, follow-up results, smoking history, clinical symptoms, CT imaging features, immunohistochemical results, and gene analysis were recorded (Table 1).

According to the literature, primary lung SRCC is most common in the stomach, and rarely occurs in the lung. Prior to our case, only 15 cases involving CT image information were recorded with an average age of 56.7 (range, 32-79) years and male to female ratio of 1.14:1.00. A history of tobacco use was obtained in only eight patients (53.3\%), and there was a lack of characteristic clinical symptoms. The incidence of lesions in the left and right lobes was scattered and most were in the periphery of the lung. Except for two patients with multiple ground glass nodules and multiple small nodules, the remaining lesions were solid, and ranged in size from 1.0 to $8 \mathrm{~cm}$. Only one patient had a cavity in the solid lesion (Figure 4). The immunohistochemistry 

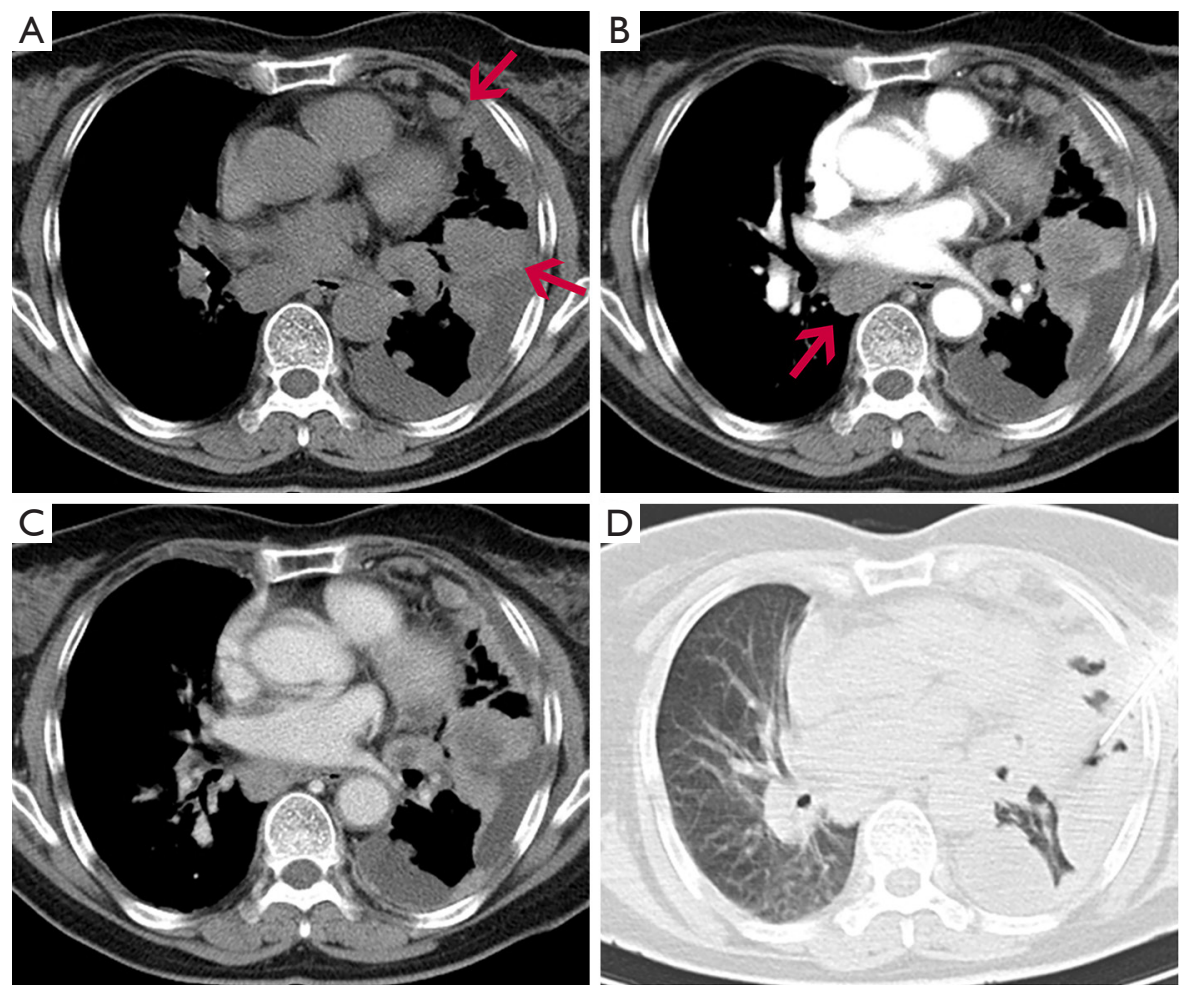

Figure 1 Chest CT scan. (A) Non-contrast enhanced CT chest image on the axial plane of the mediastinal window shows multiple nodules of different sizes and mass like soft tissue density (red arrow) in the left upper lobe, lower lobe, and under the pleura, with rough edges, uneven density, and pleural traction. (B) In the arterial phase, the soft tissue mass shows obvious enhancement, and an irregular lowdensity area can be seen in the center of the main lesion. Enlarged lymph nodes (red arrow) can be seen in the mediastinum (right posterior of left atrium). (C) Axial enhanced CT image in the venous phase. (D) Schematic diagram of the CT guided lung biopsy. CT, computed tomography.

(IHC) of primary SRCC was studied and demonstrated that 13 cases including thyroid transcription factor-1 (TTF-1) were positive, napsin A (3/4) was positive, and the positive rate was only $75 \%$. Twelve cases of CK7 were positive, and 12 cases of CK20 and six cases of CDX2 were negative (Table 2).

\section{Discussion}

Primary lung SRCC is a rare subtype of lung adenocarcinoma. In a large retrospective study $\mathrm{Ou}$ et al. found the incidence of primary SRCC accounted for about $0.5 \%$ of all lung adenocarcinoma (8), and of 50,351 patients, only 262 had primary lung SRCC. When compared with patients with lung adenocarcinoma, those with primary lung SRCC tended to be non-smokers, had a lower age of onset, a higher proportion of distant metastases, a shorter overall survival (OS), and most cases were diagnosed as stage IV (49.2\%). In 2018, Wu et al. (3) reviewed the clinicopathological and prognostic parameters of 738 patients with primary lung SRCC from the Surveillance, Epidemiology, and End Results (SEER) database, and found that most were poorly differentiated, with distant metastasis at initial diagnosis, and fewer patients received aggressive treatment. In addition, the 5-year tumor-specific survival (TSS) was only $11 \%$, with a median cancer-specific survival (CSS) of 6 months, suggesting a poor prognosis for most patients. According to the results of $\mathrm{Wu}$ et al., the survival outcome of SRCC were significantly different based on the primary sites. As compared to gastric SRCC, primary pancreatic, lung, esophageal, and gallbladder SRCC have worse prognosis, while breast and colorectal SRCC have favorable prognosis, the primary tumor location can be used as an independent prognostic factor of survival. Therefore, it is very important to distinguish SRCC from other lung adenocarcinoma and metastatic SRCC at an early stage. 

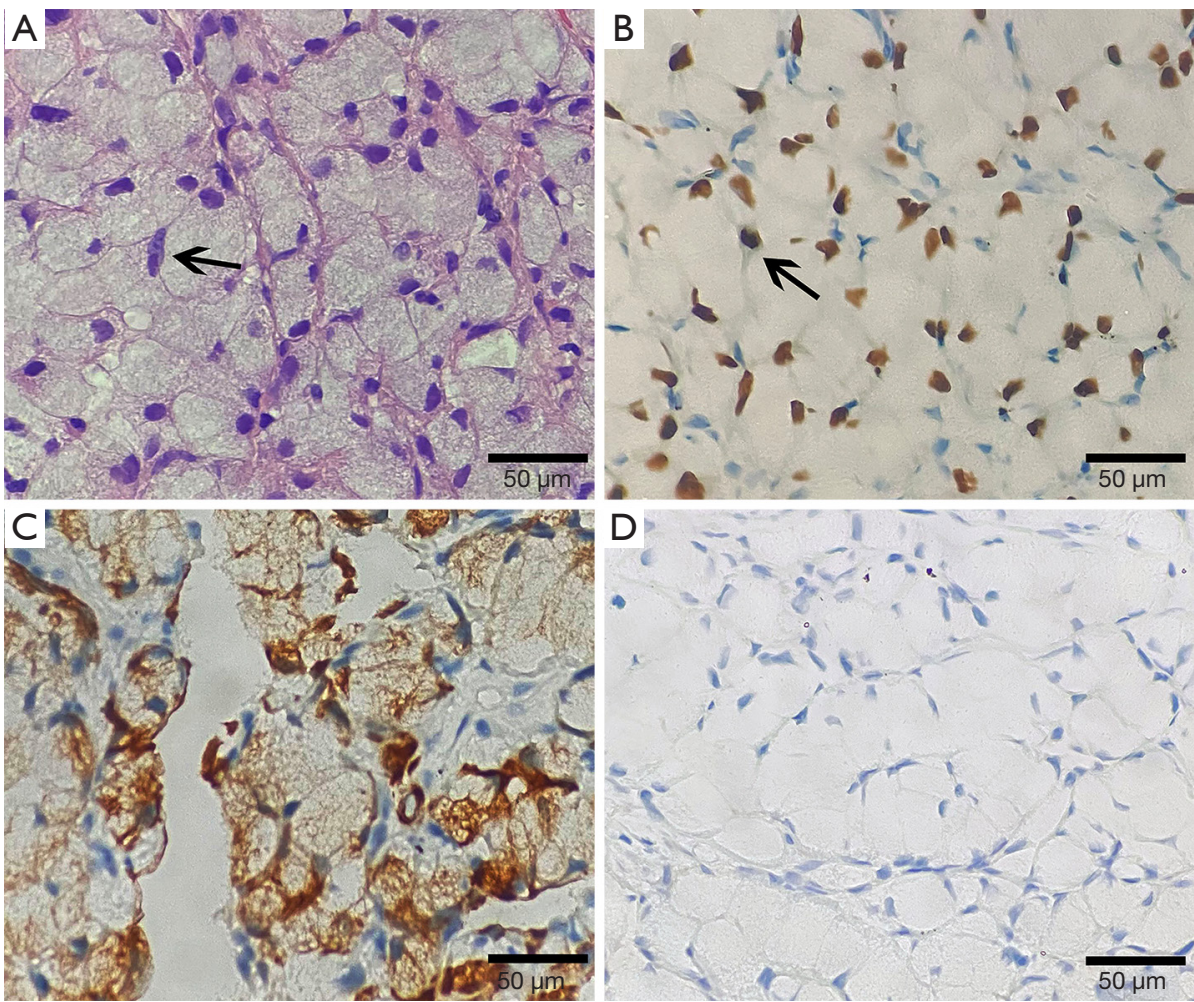

Figure 2 Primary SRCC of the lung. (A) H\&E staining (400x); showing SRCs with abundant intracytoplasmic vacuoles and peripherally displaced nuclei (arrows). (B) IHC staining (400x); TTF-1 nuclear strong positive staining (arrows). (C) IHC staining (400x); abundant cytoplasmic and membranous positivity for CK7. (D) IHC staining (400x); no reaction with cytokeratin 20 in the same tumor cells. SRCC, signet ring cell carcinoma; H\&E, hematoxylin and eosin; IHC, immunohistochemistry; TTF-1, thyroid transcription factor-1.

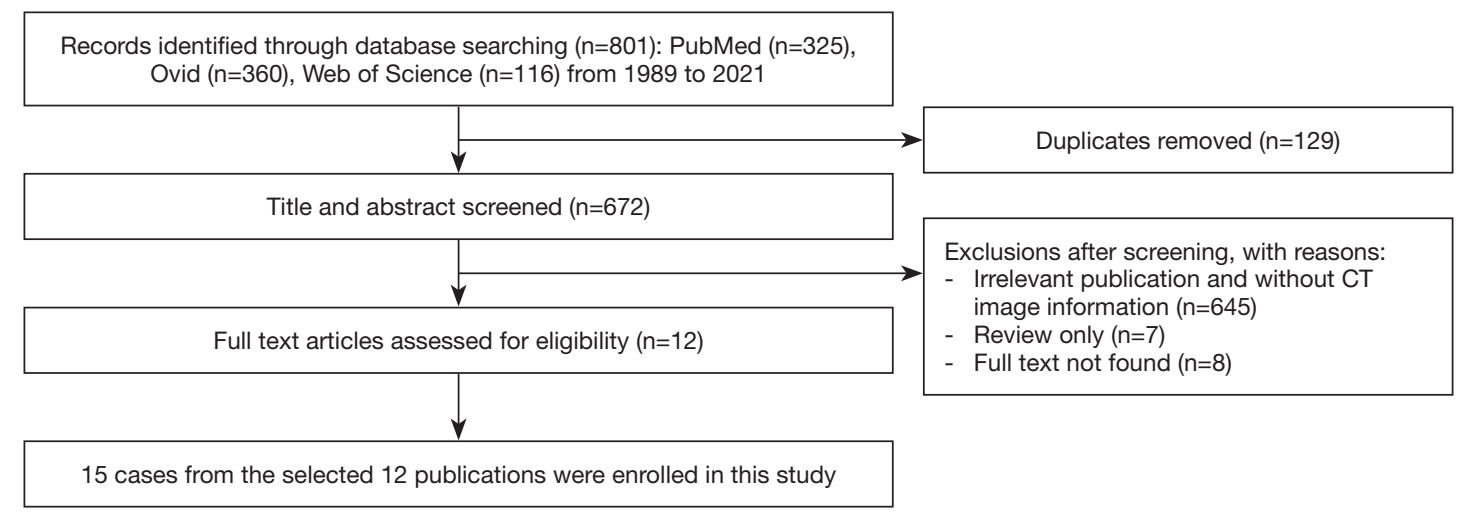

Figure 3 Flow chart of the literature screening process for primary SRCC of the lung. SRCC, signet ring cell carcinoma; CT, computed tomography. 
Table 1 Clinical and Imaging features of the cases of primary lung SRCC from the literature review

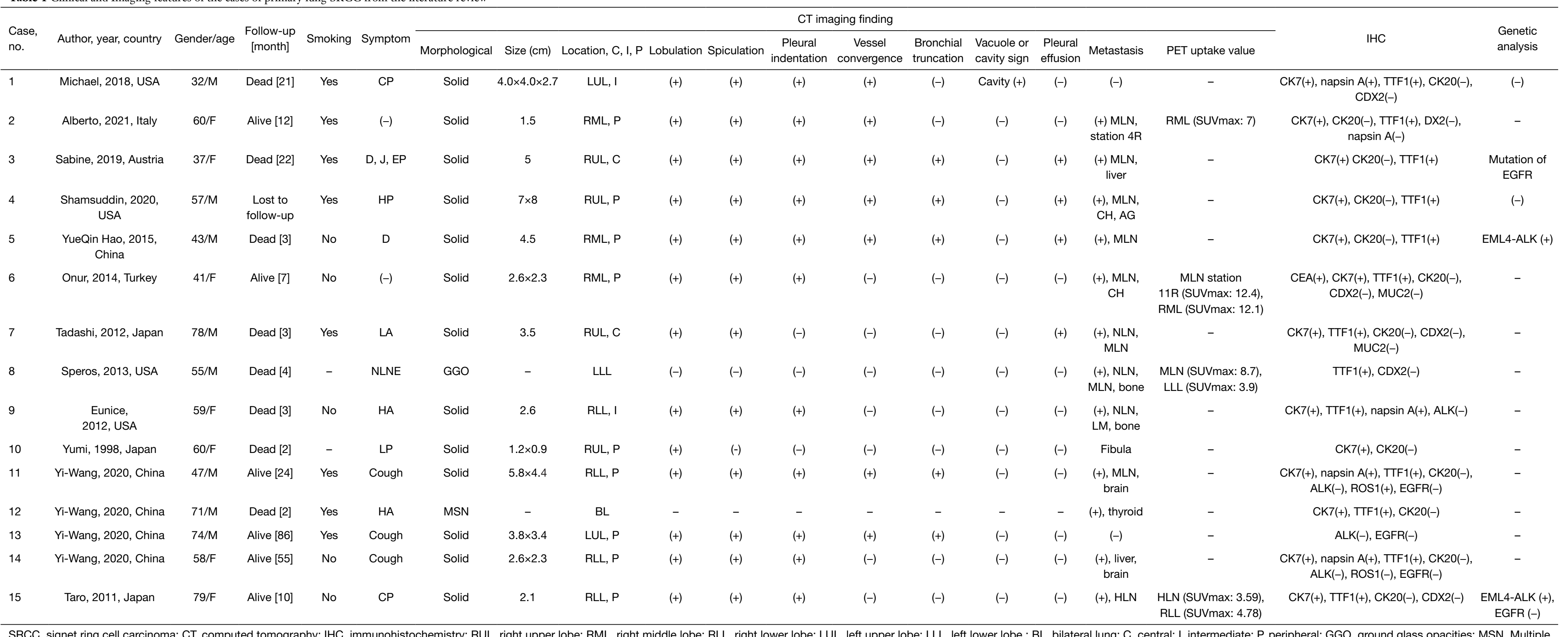

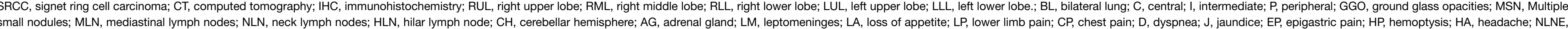
neck lymph nodes enlargement; -, not provided; (-), negative; (+) positive. 


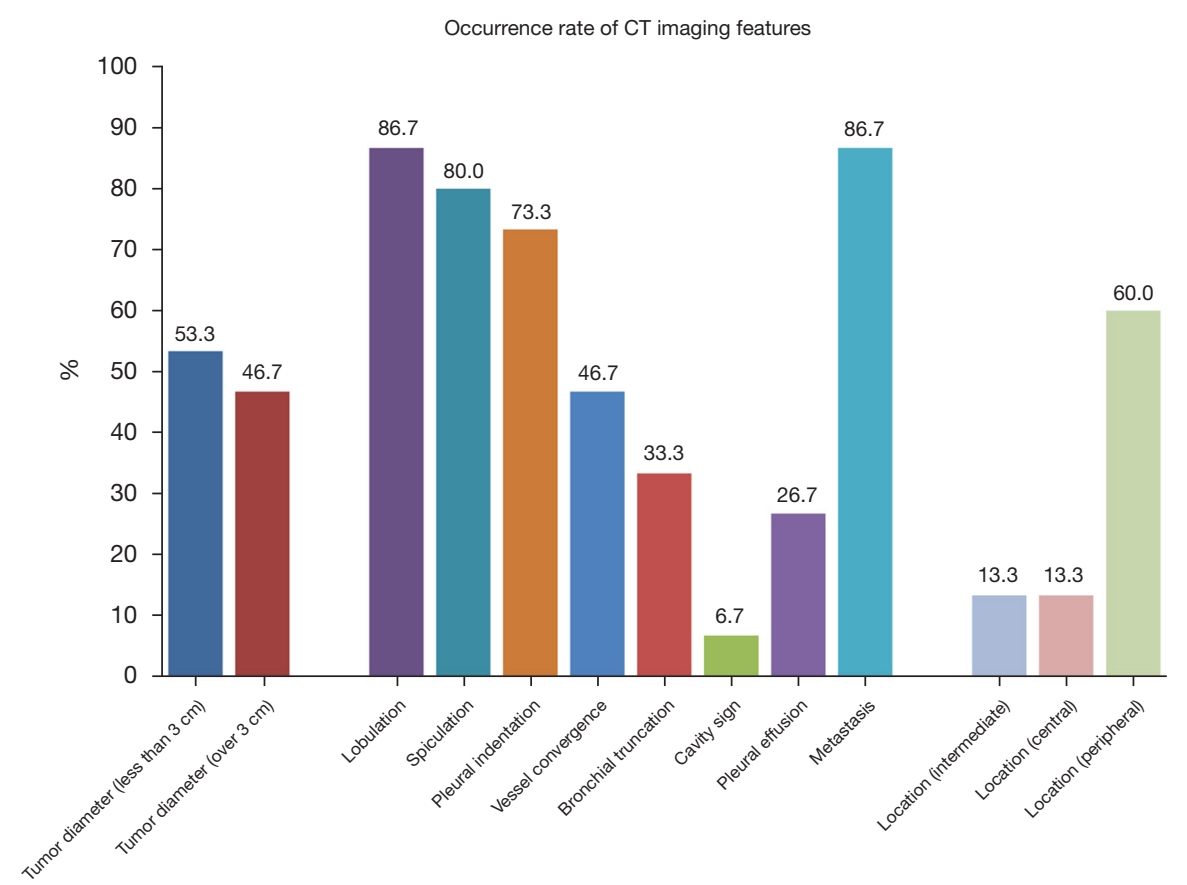

Figure 4 Imaging features of primary SRCC of the lung. The majority of tumors are solid nodules. There is no significant difference between lesions larger than or less than $3 \mathrm{~cm}$. The tumor tends to found in the peripheral lung. The masses are characterized by lobulation, spiculation, and pleural indentation, rarely forming cavities. Mediastinal lymph nodes are prone to metastasis. SRCC, signet ring cell carcinoma; CT, computed tomography.

Table 2 Positive immunohistochemical staining for CK7, CK20, TTF-1, napsin A, and CDX2 in primary SRCC of the lung. All 13 cases including TTF-1 were positive, napsin A (3/4) was positive, and the positive rate was only $75 \% ; 12$ cases of CK 7 were positive, 12 cases of CK20 and 6 cases of CDX2 were negative

\begin{tabular}{lcc}
\hline Marker & Positive cases & Percent \\
\hline Cytokeratin 7 & 12 of 12 & 100 \\
Cytokeratin 20 & 0 of 12 & 0 \\
TTF-1 & 13 of 13 & 100 \\
Napsin A & 3 of 4 & 75 \\
CDX2 & 0 of 6 & 0 \\
\hline
\end{tabular}

SRCC, signet ring cell carcinoma; TTF-1, thyroid transcription factor-1.

The clinical symptoms of primary SRCC of the lung are like those of other types of lung cancer and lack characteristic manifestations in the early stage. The main clinical manifestations are persistent irritant cough, sputum, chest tightness, hemoptysis, fever, and chest pain, although some patients remain asymptomatic for a long duration (9).
Hemoptysis rarely occurs in the clinical manifestations of collected cases of primary SRCC of the lung. Once lifethreatening hemoptysis is found, it will be very dangerous. In an emergency, bronchoscopy and argon plasma coagulation (APC) cauterization can be used to control bleeding (4).

The imaging findings of primary lung SRCC are likely to occur at the periphery of the lung, where it presents as a circular mass with unclear boundaries, solid nodule. Large tumors may have necrosis, but do not easily form cavities, and an associated pleural effusion is common. On occasion, the tumor can spread widely into the pleural cavity where, like malignant mesothelioma, it can be covered between two pleural layers, and can also be accompanied by mediastinal and hilar lymph node enlargement. Differentiation from other types of lung cancer, metastatic tumors, and malignant mesothelioma is important.

A definitive diagnosis of primary lung SRCC is determined by biopsy, histopathological examination, and immunohistochemical staining. The histopathological features show typical round or oval SRCs with clear cell boundaries, which are not clear when squeezed into each other in sheets. The cytoplasm contains abundant mucus, 
which pushes the nucleus under one side of the cell membrane forming a "half-moon" shape, and the nuclear deviations give the cells a signet ring like appearance. The diagnostic criteria are not firmly established, and the SRC component used to define primary SRCC of the lung has varied from $5 \%$ to $50 \%$ in prior reports. In the 39 study cases of Tsuta et al. (5), the SRCC component was seen as part of an adenocarcinoma in 36 cases (acinar, 27 cases; mixed bronchioloalveolar and acinar, 9 cases) and as part of an adenosquamous cell carcinoma in 2 cases; one lesion consisted of a pure SRCC. SRCC of the lung is usually a focal form related to other tissue subtype adenocarcinoma. Morphological evaluation is insufficient for the diagnosis of the disease. Further diagnosis requires deeper examination of tissue blocks. Thus, auxiliary molecular tests, such as immunohistochemical staining, are needed to determine the specific protein expression of the disease. In detailing the immunohistochemical characteristics of the disease, Merchant et al. (10) found that TTF-1 was highly expressed in primary lung SRCC, while it was not expressed in SRCC of other sites. CK7(+)/CK20(-) was also found in $94.1 \%$ of patients with primary lung SRCC. Therefore, detection of TTF-1, CK7, and CK20 is considered helpful in differential diagnosis. Since the lung is the most common metastatic organ for tumors, the possibility of a tumor originating elsewhere should be first ruled out when determining whether the lung is the primary site. Antibodies such as Villin, CDX2 (gastrointestinal tract), estrogen receptor, progesterone receptor, GCDFP-15 (mammary gland), and PSA and PSAP (prostate gland) can be used to assist diagnosis (11). At the same time, the precise diagnosis of primary SRCC needs to be combined with thorough clinical and imaging examination to rule out the possibility of occult primary metastasis.

Fluorescence in situ hybridization (FISH) in primary lung SRCC often shows $A L K$ gene translocation with EML4-ALK fusion and deletion of EGFR and KRAS mutations. EML4-ALK has become a novel therapeutic target for the treatment of primary lung SRCC, as tumors with EML4-ALK translocations may respond to $A L K$ inhibitors Crizotinib and $K$-ras with immune checkpoint inhibitors such as Pembrolizumab (12). The use of nextgeneration sequence tests to drive mutations, especially in advanced non-small cell lung cancer (NSCLC), is also increasingly a standard part of diagnostic tests, as it can detect a variety of driver mutations, including EGFR, $A L K, R O S-1$, and provide useful information for selecting chemotherapy and median age of survival (13).
Surgical treatment should be the gold standard for patients with primary lung SRCC without distant metastasis. However, when metastasis are present, aggressive treatment is also necessary, requiring multidisciplinary treatment by radiologists, surgeons, radiation oncologists, pathologists, and medical oncologists to improve the survival and quality of life of patients (12).

\section{Several issues regarding to the diagnosis for further discussion}

\section{Question 1}

The diagnosis of primary SRCC of the lung is still difficult. Usually, it depends on pathological diagnosis. At the same time, it also needs to accurately exclude whether there are primary tumors in other predilection sites, and whether there are standardized diagnostic basis and process?

\section{Expert opinion 1: Dr. Giulia M. Stella}

Despite relevant progresses some malignant entities are still obscure and, in some cases, for instance, their own origin cannot be easily identified. In other words, in some cases it is not possible to understand if a neoplastic mass in the lungs truly originates from the lungs. These enigmatic cases, representing about $3 \%$ of all cancer diagnosis, are defined as cancers of unknown primary (CUP) and represent a constant challenge in both clinical and translational oncology. Growing amount of data are available regarding the biologic landscape of CUP as well as novel techniques to identify the putative site of organ, and many results will be available in the next few years. Those finding will help to both clarify pathogenic mechanisms and to identify actionable therapeutic targets (14-18).

\section{Expert opinion 2: Dr. Sang-Won Um}

The true entity of primary SRCC of the lung is not certain at this time. It is very important to separate true SRC features from morphologic mimics such as cytoplasmic vacuolization, clear cell change and degenerative changes like tumor cell ballooning. SRC features may include heterogeneous population of lung adenocarcinomas with various driver mutations. $2015 \mathrm{WHO}$ classification of lung tumors did not acknowledge SRC as one subtype of lung adenocarcinomas (19).

\section{Expert opinion 3: Dr. Yasmeen K. Tandon}

Agree, it is a very difficult diagnosis to make prospectively given the nonspecific image findings and it really depends on pathologic diagnosis. I would say something like "Given the rarity of this entity, to the best of our knowledge, there is no standardized staging algorithm when this diagnosis 
is made". However, given that this entity more commonly occurs in other sites rather than the lung, CT abdomen/ pelvis and mammography may be considered to ensure that the biopsied lesion is in fact from a lung primary rather than representing a metastasis.

\section{Question 2}

How to choose the best treatment strategy for primary lung SRCC? How to treat the lung SRCC with different stages without gene mutation (especially in the middle and late stage) is the best treatment?

\section{Expert opinion 1: Dr. Giulia M. Stella}

Being a rare lung tumor, in case of wild type, genetic asset the treatment will be based on conventional chemotherapy (based on platinum-derived compounds and taxanes) in advanced disease stages. In those settings, radiation therapy has only palliative purposes (20). Early diagnoses of disease allows better prognosis obtained through surgery and radiotherapy treatments (11).

\section{Expert opinion 2: Dr. Sang-Won Um}

Early stage (I or II) could be treated by surgery or radiation therapy. Stage III could be treated with concurrent chemoradiation therapy \pm durvalumab according to the status of $P D-L 1$ expression. Stage IV with known driver mutation (ALK, ROS1, EGFR, or KRAS G12C) could be treated with target agents. Stage IV without known driver mutation could be treated with palliative platinum-based doublet chemotherapy \pm immune check point inhibitors according to status of $P D-L 1$ expression.

\section{Expert opinion 3: Dr. Yasmeen K. Tandon}

In my opinion, I would not expand any more on treatment than what is already written in paper. Given, its rarity, I don't think there is good evidence on the best treatment strategy at this time (which varies depending on the location) other than surgical resection in cases where there are no metastasis.

\section{Question 3}

Is there any consensus on what percentage of SRC component within adenocarcinoma defines NSCLC as primary SRC of the lung? Does the proportion of SRC components in primary SRCC affect the OS?

\section{Expert opinion 1: Dr. Giulia M. Stella}

There is, till now, no consensus on what percentage of SRC component within adenocarcinoma defines NSCLC as primary SRC of the lung (5). In tumors aroused in the stomach, the proportion of SRCs directly correlates with response to preoperative chemoradiation (21).

\section{Expert opinion 2: Dr. Sang-Won Um}

There was no definite guideline for the percentage of SRC component for the diagnosis of primary SRC of the lung. There was one report which use a cut-off value of $10 \%$ of SRC features as a diagnostic criteria (22). For the prognosis of primary SRC of the lung, the data of pre-targeted therapy era suggested the poor OS. However, more data is needed for the lung adenocarcinomas with SRC feature which had driver mutations and received the targeted therapy since there has been lots of evidence which supports good prognosis of the subjects with ALK or ROS1 fusion who received target agents.

\section{Expert opinion 3: Dr. Yasmeen K. Tandon}

From the reading I have done, there is no consensus on what percentage of SRC component within adenocarcinoma defines NSCLC as primary SRC of the lung. According to Ou et al. (8), "World Health Organization generally defines a histologic subtype of adenocarcinoma as at least $10 \%$ of the tumor". Reviewing published literature to date, various reports have used a SRC component ranging from 5\% (5), $10 \%(1,23), 30 \%(10), 75 \%(9)$ as a significant cutoff.

From the literature search I did, I can't find any source which describes if the proportion of SRC components in primary lung SRCC affect the OS. However, there is some correlation with treatment and proportion of signet cell described in the literature in gastric cancer (21).

\section{Conclusions}

Primary SRCC is a rare subtype of adenocarcinoma which can occur in various organs, including the stomach, colon, esophagus, bladder, prostate, pancreas, and breast. The vast majority of SRCCs found in the lungs are metastatic, and the correct differentiation between primary and metastatic SRCCs requires a combination of imaging examination, pathological biopsy, appropriate immunohistochemical staining, and identification of gene mutations for comprehensive analysis and diagnosis.

\section{Acknowledgments}

The authors appreciate the academic support from the AME Lung Cancer Collaborative Group. Funding: None.

\section{Footnote}

Reporting Checklist: The authors have completed the 
CARE reporting checklist. Available at https://dx.doi. org/10.21037/tlcr-21-654

Conflicts of Interest: All authors have completed the ICMJE uniform disclosure form (available at https://dx.doi. org/10.21037/tlcr-21-654). The authors have no conflicts of interest to declare.

Ethical Statement: The authors are accountable for all aspects of the work in ensuring that questions related to the accuracy or integrity of any part of the work are appropriately investigated and resolved. All procedures performed in studies involving human participants were in accordance with the ethical standards of the institutional and/or national research committee(s) and with the Helsinki Declaration (as revised in 2013). Written informed consent was obtained from the patient for publication of this case report and accompanying images. A copy of the written consent is available for review by the editorial office of this journal.

Open Access Statement: This is an Open Access article distributed in accordance with the Creative Commons Attribution-NonCommercial-NoDerivs 4.0 International License (CC BY-NC-ND 4.0), which permits the noncommercial replication and distribution of the article with the strict proviso that no changes or edits are made and the original work is properly cited (including links to both the formal publication through the relevant DOI and the license). See: https://creativecommons.org/licenses/by-nc-nd/4.0/.

\section{References}

1. Kish JK, Ro JY, Ayala AG, et al. Primary mucinous adenocarcinoma of the lung with signet-ring cells: a histochemical comparison with signet-ring cell carcinomas of other sites. Hum Pathol 1989;20:1097-102.

2. Jin N, Nguyen C, Shuja S, et al. Cytomorphology of primary signet ring-cell carcinoma of the lung. Diagn Cytopathol 2009;37:146-9.

3. Wu SG, Chen XT, Zhang WW, et al. Survival in signet ring cell carcinoma varies based on primary tumor location: a Surveillance, Epidemiology, and End Results database analysis. Expert Rev Gastroenterol Hepatol 2018;12:209-14.

4. Anwar S, Acharya S, Elsayegh D, et al. Life threatening haemoptysis in primary lung cancer-signet ring cell carcinoma. Respir Med Case Rep 2020;31:101195.
5. Tsuta K, Ishii G, Yoh K, et al. Primary lung carcinoma with signet-ring cell carcinoma components: clinicopathological analysis of 39 cases. Am J Surg Pathol 2004;28:868-74.

6. Cai Y, Xie Y, Xiong Y, et al. Clinicopathological characteristics and survival in lung signet ring cell carcinoma: a population-based study. Bosn J Basic Med Sci 2021. [Epub ahead of print]. doi: 10.17305/ bjbms.2020.5454.

7. Iwasaki T, Ohta M, Lefor AT, et al. Signet-ring cell carcinoma component in primary lung adenocarcinoma: potential prognostic factor. Histopathology 2008;52:639-40.

8. Ou SH, Ziogas A, Zell JA. Primary signet-ring carcinoma (SRC) of the lung: a population-based epidemiologic study of 262 cases with comparison to adenocarcinoma of the lung. J Thorac Oncol 2010;5:420-7.

9. Castro CY, Moran CA, Flieder DG, et al. Primary signet ring cell adenocarcinomas of the lung: a clinicopathological study of 15 cases. Histopathology 2001;39:397-401.

10. Merchant SH, Amin MB, Tamboli P, et al. Primary signetring cell carcinoma of lung: immunohistochemical study and comparison with non-pulmonary signet-ring cell carcinomas. Am J Surg Pathol 2001;25:1515-9.

11. Wang Y, Wang Y, Li J, et al. Primary signet-ring cell carcinoma of the lung: a report of seven cases. Thorac Cancer 2020;11:3015-9.

12. Adderley H, Blackhall FH, Lindsay CR. KRAS-mutant non-small cell lung cancer: Converging small molecules and immune checkpoint inhibition. EBioMedicine 2019;41:711-6.

13. Takeda M, Sakai K, Takahama T, et al. New era for next-generation sequencing in Japan. Cancers (Basel) 2019;11:742.

14. Benvenuti S, Milan M, Geuna E, et al. Cancer of Unknown Primary (CUP): genetic evidence for a novel nosological entity? A case report. EMBO Mol Med 2020;12:e11756.

15. Moran S, Martínez-Cardús A, Sayols S, et al. Epigenetic profiling to classify cancer of unknown primary: a multicentre, retrospective analysis. Lancet Oncol 2016;17:1386-95.

16. Stella GM, Kolling S, Benvenuti S, et al. Lung-seeking metastases. Cancers (Basel) 2019;11:1010.

17. Stella GM, Benvenuti S, Gentile A, et al. MET activation and physical dynamics of the metastatic process: the paradigm of cancers of unknown primary origin. EBioMedicine 2017;24:34-42.

18. Stella GM, Benvenuti S, Gramaglia D, et al. MET mutations in cancers of unknown primary origin (CUPs). 
Hum Mutat 2011;32:44-50.

19. Travis WD, Brambilla E, Nicholson AG, et al. The 2015 World Health Organization classification of lung tumors: impact of genetic, clinical and radiologic advances since the 2004 classification. J Thorac Oncol 2015;10:1243-60.

20. Wen J, Xu X, Chen J, et al. Palliative radiation therapy for metastatic primary signet ring cell carcinoma of the lung: a SEER analysis. Int J Radiat Oncol Biol Phys 2018;102:e667.

21. Charalampakis N, Nogueras González GM, Elimova $\mathrm{E}$, et al. The proportion of signet ring cell component

Cite this article as: Yang M, Yang Y, Chen J, Stella GM, Um SW, Tandon YK, Liu H. A case report of primary signet ring cell carcinoma of the lung: imaging study and literature review. Transl Lung Cancer Res 2021;10(9):3840-3849. doi: 10.21037/tlcr-21-654 in patients with localized gastric adenocarcinoma correlates with the degree of response to pre-operative chemoradiation. Oncology 2016;90:239-47.

22. Boland JM, Wampfler JA, Jang JS, et al. Pulmonary adenocarcinoma with signet ring cell features: a comprehensive study from 3 distinct patient cohorts. Am J Surg Pathol 2014;38:1681-8.

23. Shaw AT, Yeap BY, Mino-Kenudson M, et al. Clinical features and outcome of patients with non-small-cell lung cancer who harbor EML4-ALK. J Clin Oncol 2009;27:4247-53. 